\title{
Recommendations for the Use of ECT in Pregnancy: Literature Review and Proposed Clinical Protocol
}

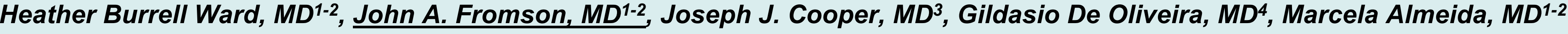 \\ 1. Brigham and Women's Hospital, Boston MA USA; 2. Harvard Medical School, Boston, MA, USA; 3. University of Chicago, Chicago, IL, USA; 4. Rhode \\ Island Hospital and Warren Alpert Medical School of Brown University, Providence, RI, USA
}

\section{BACKGROUND}

Psychiatric disorders are common in pregnancy, affecting $15-29 \%$ of pregnant women. $^{1} \quad$ Untreated depression has negative health consequences for mother and fetus. Electroconvulsive Therapy (ECT) is an effective option for the treatment of severe depression, high suicide risk, catatonia, medication-resistant illness, psychotic agitation, severe physical decline, and other life-threatening conditions.

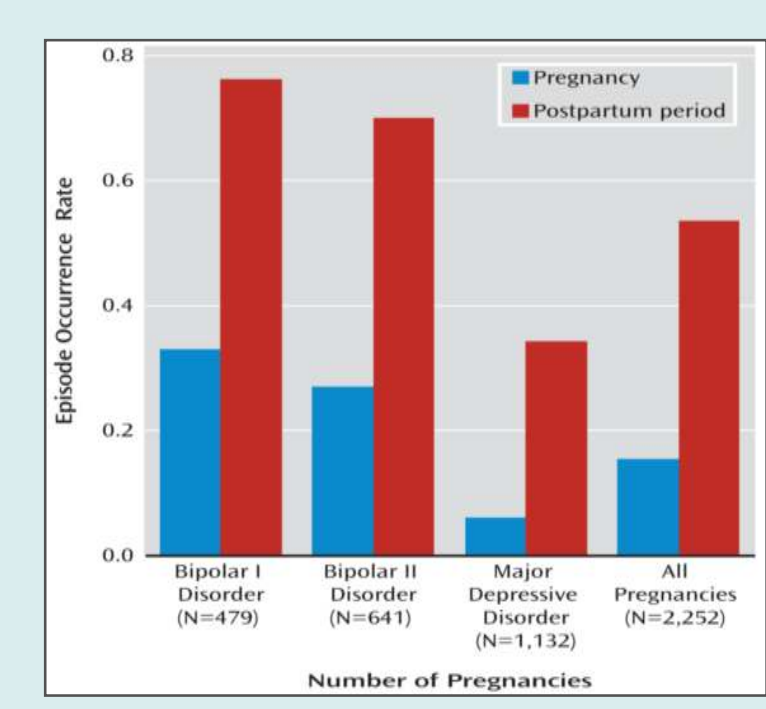

\section{METHOD}

A comprehensive review of the relevant literature regarding both ECT and psychotropic medications in pregnancy was performed, including meta-analyses of randomized controlled trials published in general medicine, anesthesiology, psychiatry and obstetrics journals and guidelines, including analyses of all 4 systematic reviews of use of ECT in pregnancy. The authors also examined the natural course, potential risks and complications from untreated mental illness in pregnancy.

\section{RESULTS}

Table 1. Complication Rates of ECT in Pregnancy

$\begin{array}{ll}\text { Miller, 19942 } & \text { Anderson and } \\ (n=300) & \text { Reti, 20073 } \\ & (n=339)\end{array}$

Leiknes, $2015^{4}$

1970- $\quad 1942-\quad$ All years $(n=115) \quad(n=54) \quad(n=169)$

Maternal

Uterine

Contractions

$2(0.6 \%)$

Premature Labor

$4(1.3 \%)$

Vaginal Bleeding

$5(1.6 \%)$

Miscarriage

Abdominal pain

$5(1.6 \%)$

Preeclampsia

Caesarian

section

Placental

Abruption

$3(1.0 \%)$

$1(0.3 \%)$

$1(0.3 \%)$

$12(3.5 \%)$

\section{4}

(30\%)

13

(28\%)

$3(7 \%) \quad 5(23 \%) \quad 8(12 \%)$

$3(7 \%) \quad 2(9 \%) \quad 5(7 \%)$

$2(4 \%) \quad 4(18 \%) \quad 6(9 \%)$

$2(4 \%)-2(3 \%)$

$9(20 \%) \quad 3(14 \%) \quad \begin{gathered}12 \\ (17 \%)\end{gathered}$

\begin{tabular}{|c|c|c|c|c|c|}
\hline Arrhythmia & $5(1.6 \%)$ & $9(2.7 \%)$ & $\begin{array}{l}13 \\
(54 \%)\end{array}$ & $2(18 \%)$ & $\begin{array}{l}15 \\
(43 \%)\end{array}$ \\
\hline Stillbirth/Death & $3(1.0 \%)$ & $1(0.3 \%)$ & $6(25 \%)$ & $2(18 \%)$ & $8(23 \%)$ \\
\hline $\begin{array}{l}\text { Fetal } \\
\text { malformations }\end{array}$ & $5(1.6 \%)$ & $1(0.3 \%)$ & $4(17 \%)$ & $3(27 \%)$ & $7(20 \%)$ \\
\hline $\begin{array}{l}\text { Respiratory } \\
\text { distress }\end{array}$ & $1(0.3 \%)$ & - & - & & $1(3 \%)$ \\
\hline Billirubinemia & - & - & $1(4 \%)$ & - & $1(3 \%)$ \\
\hline $\begin{array}{l}\text { Mental } \\
\text { impairment }\end{array}$ & $4(1.3 \%)$ & - & - & $2(18 \%)$ & $2(5 \%)$ \\
\hline
\end{tabular}

\section{CONCLUSIONS}

ECT is safe and effective for management of many psychiatric disorders in pregnancy. It allows for treatment while minimizing potential adverse effects on mother and fetus associated with pharmacotherapy. Based on changes in physiology that occur during pregnancy, we considered recommendations for treatment modifications in performing ECT in pregnancy and consolidated them into a protocol that can assist clinicians in improving access and safety of ECT for pregnant patients.

\section{Summary of Recommendations for ECT in Pregnancy}

Initial Evaluation

- Provide psychoeducation to staff to reinforce the safety and effectiveness Ensure facility has capability to treat an obstetric or neonatal complication

\section{Evaluation for ECT}

- Consult Obstetrics for Pelvic Exam

- Special consideration for risk factors for spontaneous abortion, preterm labor, abruption, and uteroplacental insufficiency

- Weekly NST of fetal well-being throughout the entire treatment course or a level 2 ultrasonogram between 18 and 22 weeks of gestational age

- If high risk for preterm labor or complications, ECT can be performed in a L\&D operating room or labor ward with a Caesarian section tray

- $\quad$ Obstetrician should be aware of the timing of the procedure

- $\quad$ Screening for psychoactive substance use disorder or signs of acute withdrawal

\section{Pre-Procedure}

- Discontinue nonessential anticholinergic medications

- Avoid NSAIDs

- $\quad$ Night before: administer $30 \mathrm{~mL} 0.3 \mathrm{M}$ sodium citrate

- NPO 8 hours prior to ECT

- $\quad 15-20 \mathrm{~min}$ before anesthesia: administer $30 \mathrm{~mL} 0.3 \mathrm{M}$ sodium citrate

- $30 \mathrm{~min}$ before: ranitidine $50 \mathrm{mg}$ or cimetidine, $+/$ - metoclopramide $20 \mathrm{mg}$

- IV hydration 12 hours prior: lactate ringer's or normal saline

- 20+ weeks: elevate right hip with foam wedge

- Fetal cardiac monitoring by Doppler ultrasound

- If fetal heart rate decreases, increase $\mathrm{O}_{2}$ and further displace uterus

- External fetal monitoring for several hours before ECT or NST with tocometer

\section{Peri-Procedure}

Continuous maternal heart rate, $\mathrm{EKG}, \mathrm{SpO}_{2}$, end-tidal $\mathrm{CO} 2$ monitoring

- If concern for contractions: use uterine tocodynamometry

- If late term or high-risk: use continuous fetal monitoring

- Consider intubation, especially after first trimester

- Preoxygenate but do not hyperventilate

- Induction: propofol $0.75-2 \mathrm{mg} / \mathrm{kg}$, methohexital $0.5-1 \mathrm{mg} / \mathrm{kg}$, or, if third trimester, consider inhalational anesthetics

- $\quad$ Paralysis: succinylcholine $0.5-1.5 \mathrm{mg} / \mathrm{kg}$ unless contraindicated

- Treat maternal hypertension (e.g. esmolol or remifentanil infusion)

- Treat maternal bradycardia, when indicated, with glycopyrrolate $2 \mathrm{mg}$

- If fetal heart rate decreases, increase $\mathrm{O}_{2}$ and further displace uterus

- If significant desaturation: get maternal $A B G$ during or after ECT

Post-Procedure

- $\quad$ Examine patient for uterine contractions, vaginal bleeding

- If contractions: treat with beta-2 adrenergic tocolytics (i.e. ritodrine)

If abdominal pain: treat with $\mathrm{IV} \mathrm{MgSO}_{4} 4 \mathrm{mg}$ diluted in $100 \mathrm{~mL}$ saline

- If contractions or vaginal bleeding: postpone further ECT

- Repeat fetal cardiac monitoring by Doppler ultrasound

- Repeat external fetal monitoring for up to several hours as needed or NST with tocometer after procedure

- If necessary: weekly fetal NSTs

- If nausea: ondansetron, metoclopramide, prochlorperazine, or meclizine

- If headache or muscle aches: treat with acetaminophen

- Anticipatory guidance: if fever $>100.4$ of or cough, decreased fetal movement, go to nearest emergency room/ obstetrical triage 